\title{
Induced Ramsey number for a star versus a fixed graph
}

\author{
Maria Axenovich \\ Department of Mathematics \\ Karlsruhe Institute of Technology \\ Germany \\ maria.aksenovich@kit.edu
}

\author{
Izolda Gorgol \\ Department of Applied Mathematics \\ Lublin University of Technology \\ Poland \\ i.gorgol@pollub.pl
}

Submitted: Feb 10, 2020; Accepted: Jan 13, 2021; Published: Mar 26, 2021

(c) The authors. Released under the CC BY-ND license (International 4.0).

\begin{abstract}
We write $F \stackrel{\text { ind }}{\longrightarrow}(H, G)$ for graphs $F, G$, and $H$, if for any coloring of the edges of $F$ in red and blue, there is either a red induced copy of $H$ or a blue induced copy of $G$. For graphs $G$ and $H$, let $\operatorname{IR}(H, G)$ be the smallest number of vertices in a graph $F$ such that $F \stackrel{\text { ind }}{\longrightarrow}(H, G)$.

In this note we consider the case when $G$ is a star on $n$ edges, for large $n$ and $H$ is a fixed graph. We prove that

$$
(\chi(H)-1) n \leqslant \operatorname{IR}\left(H, K_{1, n}\right) \leqslant(\chi(H)-1)^{2} n+\epsilon n,
$$

for any $\epsilon>0$, sufficiently large $n$, and $\chi(H)$ denoting the chromatic number of $H$. The lower bound is asymptotically tight for any fixed bipartite $H$. The upper bound is attained up to a constant factor, for example when $H$ is a clique.
\end{abstract}

Mathematics Subject Classifications: 05C55, 05D10

\section{Introduction}

We write $F \stackrel{\text { ind }}{\longrightarrow}(H, G)$ for graphs $F, G$, and $H$, if for any coloring of the edges of $F$ in red and blue, there is either a red induced copy of $H$ or a blue induced copy of $G$. For graphs $G$ and $H$, the induced Ramsey number for $H$ and $G$, denoted by $\operatorname{IR}(H, G)$, is the smallest number of vertices in a graph $F$ such that $F \stackrel{\text { ind }}{\longrightarrow}(H, G)$. The existence of such a graph $F$ for any graphs $H$ and $G$ was first proven by Deuber [4], extending a classical result by Ramsey [17]. This led to extensive research on induced Ramsey numbers. For more recent results, see papers of Conlon, Fox, and Sudakov [3, 7], Dudek, Frankl, and Rödl [5], as well as Kostochka and Sheikh [14], Schaefer and Shah [18], and Kohayakawa, 
Prömel, and Rödl [13]. We do not attempt to mention the numerous results on induced hypergraph Ramsey numbers here.

In this note we consider the case when $G$ is a star on $n$ edges, i.e., $G=K_{1, n}$, and $H$ is a fixed graph.

Finding the classical Ramsey number of a graph $H$ versus a star, $R\left(H, K_{1, n}\right)$, translates into finding graphs with maximum degree less than $n$ and having no $H$ in the complement, i.e., the so-called min-degree extremal problem for $H$. The corresponding induced Ramsey problem has a very different nature. It becomes nontrivial already when $H$ is a matching. Specifically $\operatorname{IR}\left(n K_{2}, K_{1, n}\right)$ is superlinear as shown by Conlon, Fox, and Sudakov [3, 6]; see also a related result by Fox, Huang, and Sudakov [8]: $n e^{c \log ^{*} n} \leqslant \operatorname{IR}\left(n K_{2}, K_{1, n}\right) \leqslant$ $n e^{c^{\prime} \sqrt{\log n}}$, where $\log ^{*} n=0$ if $n \leqslant 1$ and $\log ^{*} n=1+\log ^{*}(\log n)$ if $n>1$.

Our focus is the case when $H$ is a fixed graph and $n$ grows. In this regime, one can easily show that $\operatorname{IR}\left(H, K_{1, n}\right)=\Theta(n)$. We provide easy general bounds $n \leqslant \operatorname{IR}\left(H, K_{1, n}\right) \leqslant$ $|E(H)|(n-1)+|V(H)|$, confirming this fact. Since $\operatorname{IR}\left(H, K_{1, n}\right) \geqslant(\chi(H)-1) n$, where $\chi(H)$ denotes the chromatic number of $H$, we see that $\operatorname{IR}\left(H, K_{1, n}\right) \geqslant 2 n$ for non-bipartite $H$. We show that for bipartite $H$, the trivial lower bound $\operatorname{IR}\left(H, K_{1, n}\right) \geqslant n$ is asymptotically tight. We also provide general bounds on $\operatorname{IR}\left(H, K_{1, n}\right)$ in terms of $\chi(H)$. Note that when $H$ is a star, the situation is quite special, namely $\operatorname{IR}\left(K_{1, \ell}, K_{1, n}\right)=n+\ell$ with $K_{1, n+\ell-1} \stackrel{\text { ind }}{\longrightarrow}\left(K_{1, \ell}, K_{1, n}\right)$. We include the proof of this fact for completeness in Lemma 4 . Here are the three main results of this paper.

Theorem 1. Let $H$ be a fixed graph that is not a star and that has no isolated vertices. There is a positive constant c such that for any sufficiently large $n, \operatorname{IR}\left(H, K_{1, n}\right) \geqslant n+c \sqrt{n}$.

Theorem 2. For any $\gamma>0$ and any graph $H$ there is $n_{0} \in \mathbb{N}$ such that for any $n>n_{0}$, $\operatorname{IR}\left(H, K_{1, n}\right) \leqslant(\chi(H)-1)^{2} n+\gamma n$.

The following gives more precise upper bounds for some bipartite graphs that allows to replace the $\gamma n$ term of Theorem 2 with a $o(n)$ term.

Theorem 3. Let $t$ be an integer, $t \geqslant 2$. There is a positive constant $c$ and $n_{0} \in \mathbb{N}$, such that for any $n>n_{0}, \operatorname{IR}\left(2 K_{2}, K_{1, n}\right) \leqslant n+c n \log ^{-1 / 4} n, \operatorname{IR}\left(P_{4}, K_{1, n}\right) \leqslant n+c n \ln ^{-1 / 4} n$, and $n+n^{1-\frac{2}{t+1}} \ln ^{-1} n \leqslant \operatorname{IR}\left(K_{t, t}, K_{1, n}\right) \leqslant n+c n^{1-\frac{1}{t+1}}$.

The paper is structured as follows. We provide some known results on related induced Ramsey numbers and give basic general bounds in Section 2. We prove our main results in Section 3. For standard graph theoretic notions and terminology, we refer the reader to West [19].

\section{Known results and general bounds}

\section{1 $\operatorname{IR}\left(H, K_{1, n}\right)$ for $H$ complete, complete bipartite, path, star, and cycle}

The known results for $\operatorname{IR}\left(H, K_{1, n}\right)$ include $\operatorname{IR}\left(K_{t}, K_{1, n}\right)=(n-1) t(t-1) / 2+t$, see Gorgol [10], $\operatorname{IR}\left(K_{t, t}, K_{1, n}\right) \leqslant(n+1) t, \operatorname{IR}\left(P_{t}, K_{1, n}\right) \leqslant(t-1) n+1, \operatorname{IR}\left(C_{t}, K_{1, n}\right) \leqslant t n$, see Gorgol 
[11]. Note that $\operatorname{IR}\left(C_{4}, K_{1, n}\right) \geqslant R\left(C_{4}, K_{1, n}\right) \geqslant n+\left\lfloor\sqrt{n}-6 n^{11 / 40}\right\rfloor$, as shown by Burr, Erdős, Faudree, Rousseau, and Schelp in 1989 [2]. The term $n^{11 / 40}$ came from the best known upper bound, at that time, on the difference between two consecutive primes. In fact, Parsons [16] earlier proved that when $q$ is a power of a prime and $n=q^{2}+1$ then $R\left(C_{4}, K_{1, n}\right)=q^{2}+q+2$. While the result for stars was announced by Harary et al. [12] in the diagonal case, the proof was not included in that paper. We state it here for completeness.

Lemma 4. For any positive integers $\ell$ and $n, \operatorname{IR}\left(K_{1, \ell}, K_{1, n}\right)=n+\ell$.

Proof. For the upper bound, observe that $K_{1, n+\ell-1} \stackrel{\text { ind }}{\longrightarrow}\left(K_{1, \ell}, K_{1, n}\right)$. For the lower bound, we claim that any graph $G$ on $k$ vertices, $k<n+\ell$ can be colored with no blue induced star on $n$ edges and no red induced star on $\ell$ edges. Note that we can assume that $\ell, n \geqslant 2$ since otherwise the result trivially holds. Let $\Delta(G)$ denote the maximum degree of $G$. If $\Delta(G)<k-1$, then by Vizing's theorem we can edge-decompose $G$ into at most $\Delta+1 \leqslant k-2+1=k-1 \leqslant n+\ell-2$ matchings. Color $n-1$ of these matchings blue and $\ell-1$ of these matchings red. This coloring contains no blue star on $n$ edges and no red star on $\ell$ edges. If $\Delta(G)=k-1$, then there is a vertex $v$ adjacent to all other vertices of $G$. Note that in this case no induced star with at least 2 edges can have $v$ as a leaf. Let $G^{\prime}$ be the graph obtained from $G$ by deleting the edges incident to $v$. Then $\Delta\left(G^{\prime}\right)<k-1$, so $G^{\prime}$ can be colored with no blue induced star on $n$ edges and no red induced star on $\ell$ edges. Now, color $n-1$ edges incident to $v$ blue and the remaining, $(k-1)-(n-1)<\ell$ edges incident to $v$ red.

\subsection{General bounds on $\operatorname{IR}\left(H, K_{1, n}\right)$}

Gorgol [9] proved that $\operatorname{IR}\left(H, K_{1, n}\right) \geqslant(n-1) \omega(\omega-1) / 2+\omega$, where $\omega=\omega(H)$ is the order of a largest clique in $H$. On the other hand, the classical lower bound $\operatorname{IR}\left(H, K_{1, n}\right) \geqslant$ $n(\chi(H)-1)+1$ holds by splitting the vertex set of any graph on $n(\chi(H)-1)$ vertices into $\chi(H)-1$ sets of equal sizes, coloring all edges inside the sets blue and all edges between the sets red. This coloring then has no red copy of $H$ and no blue copy of $K_{1, n}$.

The following easy lemma generalizes the upper bound on $\operatorname{IR}\left(H, K_{1,2}\right)$ by Kostochka and Sheikh [14]. While this bound is much weaker than our general upper bound, we include it here since it holds for any $n$ and does not use the Regularity Lemma. So, it is more applicable for bounding induced Ramsey numbers of fixed small graphs.

For a graph $H$ with vertices $v_{1}, \ldots, v_{n}, a$ blow-up of $H$ is a graph whose vertex set is a pairwise disjoint union of sets $V_{1}, \ldots, V_{n}$, called the parts of the blow-up and two vertices adjacent if and only if they are from sets $V_{i}$ and $V_{j}$ for some $i \neq j$ such that $v_{i} v_{j}$ is an edge in $H$.

Lemma 5. Let $H$ be a graph, then $\operatorname{IR}\left(H, K_{1, n}\right) \leqslant|E(H)|(n-1)+|V(H)|$. If $H^{\prime}$ is a blow-up of $H$ with s vertices in each part, then $\operatorname{IR}\left(H^{\prime}, K_{1, n}\right) \leqslant s(|E(H)|(n-1)+|V(H)|)$.

Proof. Let the vertices of $H$ be $v_{1}, \ldots, v_{k}$ such that $v_{i}$ has $d_{i}$ neighbours among $v_{1}, \ldots, v_{i-1}$, for $i=2, \ldots, k$. Then in particular $d_{2}+\cdots+d_{k}=|E(H)|$. Consider $G$ a blow-up of $H$ with 
the vertex set being a union of pairwise disjoint sets $V_{1}, \ldots, V_{k}$ (representing $v_{1}, \ldots, v_{k}$, respectively), with $\left|V_{1}\right|=1,\left|V_{i}\right|=(n-1) d_{i}+1, i=2, \ldots, k$. We see that any induced subgraph of $G$, formed by choosing a single vertex from each set $V_{i}$ is isomorphic to $H$. We claim that $G \stackrel{\text { ind }}{\longrightarrow}\left(H, K_{1, n}\right)$ in a stronger form, i.e., such that any red/blue edge coloring of $G$ contains either a blue star on $n$ edges or a red induced copy of $H$ with respective vertices $u_{1}, \ldots, u_{k}$ in the corresponding sets $V_{1}, \ldots, V_{k}$. Consider a red/blue coloring of $G$ with no blue star on $n$ edges. Let $u_{1}$ be from $V_{1}$. Assume we found a red induced copy of $H_{i-1}$ of $H\left[\left\{v_{1}, \ldots, v_{i-1}\right\}\right]$ with vertices $u_{j} \in V_{j}, j=1, \ldots, i-1$, corresponding to the $v_{j}$ 's. Consider $V_{i}$. There are $d_{i}$ edges from $v_{i}$ to $\left\{v_{1}, \ldots, v_{i-1}\right\}$ in $H$, so there are at most $(n-1) d_{i}$ vertices of $V_{i}$ that send a blue edge to some of $u_{1}, \ldots, u_{i-1}$. Since $\left|V_{i}\right|=(n-1) d_{i}+1$, there is a vertex in $V_{i}$ that sends only red edges to $\left\{u_{1}, \ldots, u_{i-1}\right\}$. Let $u_{i}$ be that vertex. Then $G\left[V\left(H_{i-1}\right) \cup\left\{u_{i}\right\}\right]$ is a red induced copy of $H\left[\left\{v_{1}, \ldots, v_{i}\right\}\right]$.

When $H^{\prime}$ is a blow-up of $H$, we proceed similarly by taking $G^{\prime}$ to be a blow-up of $H$ with parts of sizes $s, s\left((n-1) d_{2}+1\right), \ldots, s\left((n-1) d_{i}+1\right), \ldots$ and embedding a part of $H^{\prime}$ corresponding to the blow-up of $v_{i}$ in $V_{i}$.

\section{Proofs of the main results}

\subsection{Proof of Theorem 1}

Theorem 6. Let $H$ be a graph that is neither empty nor complete bipartite. Then $\operatorname{IR}\left(H, K_{1, n}\right) \geqslant n+q+1$, for $q=\sqrt{n+1 / 4}-1 / 2$.

Proof. Consider a graph $G$ on $n+q$ vertices. We shall show that we can color its edges such that the red graph is complete bipartite and there is no blue induced star on $n$ edges. We say that a star is large if it has at least $n$ edges and is induced. We call a vertex large if it is a center of a large star. Consider all maximal large stars. Let $X$ be the set of centers of these stars.

Let $Y$ be a set of leaves of a large star such that $|Y|=n$. Note first that no vertex in $Y$ is large because $Y$ is an independent set, so its members have neighbors only in $V(G)-Y$, a set of size at most $n+q-n<n$. Thus $X \cap Y=\emptyset$ and thus there are at most $q$ large vertices, i.e., $|X| \leqslant q$.

Consider the bipartite subgraph of $G$ with parts $X$ and $Y$. We claim that all but at most $q^{2}$ vertices of $Y$ send $|X|$ edges to $X$. Let $e$ be the number of edges between $X$ and $Y$. Let $\ell$ be the number of vertices in $Y$ that send at most $|X|-1$ edges to $X$. Then on the one hand we have that $e \geqslant|X|(n-q)$ since each large vertex has at least $n$ neighbors, at most $q$ of those outside of $Y$. On the other hand $e \leqslant \ell(|X|-1)+(n-\ell)|X|$. Setting these two inequalities together, we have that $|X|(n-q) \leqslant \ell(|X|-1)+(n-\ell)|X|$. Thus $\ell \leqslant|X| q \leqslant q^{2}$.

Thus there is a set $Y^{\prime} \subseteq Y$, so that $Y^{\prime}, X$ form a complete bipartite graph and $\left|Y^{\prime}\right| \geqslant n-q^{2} \geqslant q$, for $q \leqslant \sqrt{n+1 / 4}-1 / 2$. Now color this complete bipartite graph with parts $X$ and $Y^{\prime}$ red, and color all remaining edges blue. Clearly there is no induced red $H$. We see that each large vertex has at least $q$ of its incident edges colored red. Thus, 
there are at most $n+q-1-q<n$ blue edges incident to a large vertex. Therefore there is no blue large star.

Proof of Theorem 1. Let $H$ be a graph that is not a star and that doesn't have isolated vertices. If $H$ is not a complete bipartite graph, Theorem 6 gives us the desired result. If $H$ is a complete bipartite graph, it contains $C_{4}$ since $H$ is not a $\operatorname{star}$. Then $\operatorname{IR}\left(H, K_{1, n}\right) \geqslant$ $n+\left\lfloor\sqrt{n}-6 n^{11 / 40}\right\rfloor$, as follows from the above mentioned result of Burr et al. [2].

\subsection{Proof of Theorem 2}

In order to prove the main result, we shall be applying a multicolored version of Szemerédi's Regularity Lemma. The following Lemma will be used to analyze the reduced graph, with pink corresponding to sparse in red regular pairs and yellow corresponding to non-regular pairs.

Lemma 7. Let $\epsilon^{\prime}>0$ be a fixed small constant, $r$ be an integer, $r \geqslant 2, C>1, \epsilon^{\prime} \ll$ $C^{-3} 6^{-r}$, and $n_{0}$ be sufficiently large. Let $G$ be an edge-colored complete r-partite graph with parts $X_{1}, \ldots, X_{r}$ each of size at least $n_{0}$, with edge colors yellow, pink, and white. Assume that the following conditions hold:

1. $\left|X_{1}\right| \geqslant\left|X_{2}\right| \geqslant \cdots \geqslant\left|X_{r}\right|$,

2. $\frac{1}{C} \leqslant \frac{\left|X_{i}\right|}{\left|X_{j}\right|} \leqslant C$ for any $i, j \in[r]$,

3. for any $i, j \in[r], 1 \leqslant j<i$, and any $v \in X_{i}$, v sends at most $\left(\frac{1}{r-1}-\frac{\epsilon^{\prime} C^{3}}{r-1}\right)\left|X_{j}\right|$ pink edges to $X_{j}$, and

4. the total number of yellow edges is at most $\epsilon^{\prime} 6^{-r} \sum_{1 \leqslant i<j \leqslant r}\left|X_{i}\right|\left|X_{j}\right|$.

Then there is a copy of $K_{r}$ in $G$ with all edges colored white.

Proof. We shall proceed by induction on $r$.

Let $r=2$. We first claim that there is a vertex $v$ in $X_{2}$ such that $v$ is incident to at most $\epsilon^{\prime} 6^{-2}\left|X_{1}\right|$ yellow edges. Indeed, otherwise the total number of yellow edges would be larger than assumed. So, we see that $v$ is incident to at most $\epsilon^{\prime} 6^{-2}\left|X_{1}\right|$ yellow edges and at most $\left(\frac{1}{1}-\epsilon^{\prime} C^{3}\right)\left|X_{1}\right|$ pink edges. Thus there are at least $\left(\left(C^{3}-6^{-2}\right) \epsilon^{\prime}\right)\left|X_{1}\right|>0$ white edges, and in particular there is a white $K_{2}$.

Assume that $r \geqslant 3$ and that the statement of the lemma holds for smaller values of $r$. We shall be using the fact that $C^{2}\left|X_{r}\right|^{2} \geqslant\left|X_{i}\right|\left|X_{j}\right| \geqslant \frac{1}{C^{2}}\left|X_{r}\right|^{2}$ for any $i, j \in[r]$. We shall find a vertex $u$ in $X_{r}$ that sends a lot of white edges to each of the $X_{i}$ 's, $i \in[r-1]$. Then we shall apply induction to the subgraph spanned by the white neighborhood of $u$.

First, we claim that there is a vertex in $X_{r}$ that sends at most $\epsilon^{\prime} 6^{-r}\left(C^{3}\left(\begin{array}{l}r \\ 2\end{array}\right)\right)\left|X_{i}\right|$ yellow edges to each $X_{i}, i \in[r-1]$. Otherwise each vertex of $X_{r}$ sends more than $\epsilon^{\prime} 6^{-r} C^{3}\left(\begin{array}{c}r \\ 2\end{array}\right)\left|X_{i}\right|$ yellow edges to some $X_{i}$, thus the total number of yellow edges between $X_{r}$ and the rest of the graph is more than 


$$
\begin{aligned}
\epsilon^{\prime} 6^{-r} C^{3}\left(\begin{array}{l}
r \\
2
\end{array}\right)\left|X_{r}\right|\left|X_{r-1}\right| & \geqslant \epsilon^{\prime} 6^{-r} C^{3}\left(\begin{array}{l}
r \\
2
\end{array}\right) \frac{1}{C}\left|X_{r}\right|^{2} \\
& \geqslant \epsilon^{\prime} 6^{-r} C^{3}\left(\begin{array}{l}
r \\
2
\end{array}\right) \frac{1}{C} \frac{1}{C^{2}} \frac{1}{\left(\begin{array}{l}
r \\
2
\end{array}\right)} \sum_{1 \leqslant i<j \leqslant r}\left|X_{i}\right|\left|X_{j}\right| \\
& =\epsilon^{\prime} 6^{-r} \sum_{1 \leqslant i<j \leqslant r}\left|X_{i}\right|\left|X_{j}\right| .
\end{aligned}
$$

This contradicts the assumption on the total number of yellow edges.

Let $u=u_{r}$ be such a vertex, i.e., a vertex from $X_{r}$ that sends at most $\epsilon^{\prime} 6^{-r} C^{3}\left(\begin{array}{c}r \\ 2\end{array}\right)\left|X_{i}\right|$ yellow edges to each $X_{i}, i \in[r-1]$. So, we know that for any $i \in[r-1]$, the number of vertices of $X_{i}$ joined to $u$ by a pink or a yellow edge is at most

$$
\left(\frac{1}{r-1}-\frac{\epsilon^{\prime} C^{3}}{r-1}+\epsilon^{\prime} 6^{-r} C^{3}\left(\begin{array}{l}
r \\
2
\end{array}\right)\right)\left|X_{i}\right| \leqslant \frac{1}{r-1}\left|X_{i}\right|
$$

Thus $u$ is joined to at least $\left(1-\frac{1}{r-1}\right)\left|X_{i}\right|$ vertices of $X_{i}$ via white edges, for each $i \in[r-1]$.

We choose a subset $X_{i}^{\prime}$ of $X_{i}$, such that $\left|X_{i}^{\prime}\right|=\left(1-\frac{1}{r-1}\right)\left|X_{i}\right|=\frac{r-2}{r-1}\left|X_{i}\right|$ and $u$ sends only white edges to $X_{i}^{\prime}$, for each $i \in[r-1]$. Let $G^{\prime}$ be a subgraph of $G$ induced by $X_{1}^{\prime}, \ldots, X_{r-1}^{\prime}$ with the inherited coloring. We shall argue that we can apply induction to $G^{\prime}$. For that we need to check that

1. $\left|X_{1}^{\prime}\right| \geqslant \ldots \geqslant\left|X_{r-1}^{\prime}\right|$,

2. $\frac{1}{C} \leqslant \frac{\left|X_{i}^{\prime}\right|}{\left|X_{j}^{\prime}\right|} \leqslant C$ for any $i, j \in[r-1]$,

3. the total number of yellow edges in $G^{\prime}$ is at most $\epsilon^{\prime} 6^{-r+1} \sum_{1 \leqslant i<j \leqslant r-1}\left|X_{i}^{\prime}\right|\left|X_{j}^{\prime}\right|$, and

4. for any $i, j \in[r-1], 1 \leqslant j<i$, each vertex $v$ in $X_{i}^{\prime}$ sends at most $\left(\frac{1}{r-2}-\frac{\epsilon^{\prime} C^{3}}{r-2}\right)\left|X_{j}^{\prime}\right|$ pink edges to $X_{j}^{\prime}$.

The first two statements follow trivially since $\left|X_{i}^{\prime}\right|=\frac{r-2}{r-1}\left|X_{i}\right|, i \in[r-1]$. Let us verify item 3. Here, we shall be using the fact that $\left|X_{r}\right| \leqslant\left|X_{i}\right|$ for any $\in[r-1]$, thus $\left|X_{r}\right| \leqslant\left(\left|X_{1}\right|+\cdots+\left|X_{r-1}\right|\right) /(r-1)$. We know that the total number of yellow edges in $G^{\prime}$ is at most the total number of yellow edges in $G$, that is

$$
\begin{aligned}
& \epsilon^{\prime} 6^{-r} \sum_{1 \leqslant i<j \leqslant r}\left|X_{i}\right|\left|X_{j}\right| \\
= & \epsilon^{\prime} 6^{-r}\left(\sum_{1 \leqslant j \leqslant r-1}\left|X_{r}\right|\left|X_{j}\right|+\sum_{1 \leqslant i<j \leqslant r-1}\left|X_{i}\right|\left|X_{j}\right|\right) \\
\leqslant & \epsilon^{\prime} 6^{-r}\left(\sum_{1 \leqslant j \leqslant r-1} \frac{\left(\left|X_{1}\right|+\cdots+\left|X_{r-1}\right|\right)}{r-1}\left|X_{j}\right|+\sum_{1 \leqslant i<j \leqslant r-1}\left|X_{i}\right|\left|X_{j}\right|\right)
\end{aligned}
$$




$$
\begin{aligned}
& =\epsilon^{\prime} 6^{-r}\left(\frac{r}{r-1} \sum_{1 \leqslant i<j \leqslant r-1}\left|X_{i}\right|\left|X_{j}\right|\right) \\
& =\epsilon^{\prime} 6^{-r}\left(\frac{r}{r-1} \frac{(r-1)^{2}}{(r-2)^{2}} \sum_{1 \leqslant i<j \leqslant r-1}\left|X_{i}^{\prime}\right|\left|X_{j}^{\prime}\right|\right) \\
& \leqslant \epsilon^{\prime} 6^{-r+1}\left(\sum_{1 \leqslant i<j \leqslant r-1}\left|X_{i}^{\prime}\right|\left|X_{j}^{\prime}\right|\right) .
\end{aligned}
$$

To verify the last inequality observe that $\frac{r(r-1)}{(r-2)^{2}} \leqslant 6$ for any $r \geqslant 3$.

Finally, lets verify item 4 . We have that the number of pink edges from a vertex $v \in X_{i}^{\prime}$ to $X_{j}^{\prime}$, for $j<i$ is at most the number of pink edges from $v$ to $X_{j}$ in $G$, that is at most

$$
\left(\frac{1}{r-1}-\frac{\epsilon^{\prime} C^{3}}{r-1}\right)\left|X_{j}\right|=\left(\frac{1}{r-1}-\frac{\epsilon^{\prime} C^{3}}{r-1}\right) \frac{r-1}{r-2}\left|X_{j}^{\prime}\right|=\left(\frac{1}{r-2}-\frac{\epsilon^{\prime} C^{3}}{r-2}\right)\left|X_{j}^{\prime}\right| .
$$

So, now, as items 1.-4. are verified, we have a white $K_{r-1}$ in $G^{\prime}$ that, together with $u$, forms a white $K_{r}$ in $G$.

Proof of Theorem 2. Consider $\gamma^{\prime}, 0<\gamma^{\prime}<1$ and let $H$ be a graph of chromatic number $r$. Let $n_{0}$ be sufficiently large. We need to show that $\operatorname{IR}\left(H, K_{1, n}\right) \leqslant(r-1)^{2} n+\gamma^{\prime} n$.

Let $\gamma=\frac{\gamma^{\prime}}{2(r-1)^{2}+1}$ and constants $\epsilon, \sigma, \eta$ be chosen such that $0<\epsilon \ll \eta \ll \sigma \ll \gamma$. Note that $\gamma<1 / 2$ for any $r \geqslant 2$. Let $Y_{1}, \ldots, Y_{r}$ be pairwise disjoint vertex sets, such that $\left|Y_{r}\right|=\gamma n$ and $\left|Y_{i}\right|=(1+2 \gamma)(r-1) n, i \in[r-1]$. Then $\sum_{1 \leqslant i \leqslant r}\left|Y_{i}\right|=$ $(r-1)^{2} n+\left(2(r-1)^{2}+1\right) \gamma n=(r-1)^{2} n+\gamma^{\prime} n$.

Let $G^{\prime}$ be a random $r$-partite graph with parts $Y_{1}, \ldots, Y_{r}$ with probability $\sigma$ of a given edge between different parts not to be selected. Then with positive probability each vertex not in a part $Y_{i}$ sends between $0.9 \sigma\left|Y_{i}\right|$ and $1.1 \sigma\left|Y_{i}\right|$ "non-edges" to $Y_{i}, i \in[r]$. In addition, with high probability there is at least a $\sigma / 2$-proportion of "non-edges" between any $\tilde{Y}_{i} \subseteq Y_{i}$ and $\tilde{Y}_{j} \subseteq Y_{j}$ with $\left|\tilde{Y}_{i}\right| \geqslant\left|Y_{i}\right| / M$ and $\left|\tilde{Y}_{j}\right| \geqslant\left|Y_{j}\right| / M$, for any distinct $i, j \in[r]$ and any constant $M$. Therefore with a positive probability there is a graph satisfying these properties. We call such a graph $G$.

Formally, let $G$ be an $r$-partite graph with parts $Y_{1}, \ldots, Y_{r}$ such that:

1. each vertex not in a part $Y_{i}$ sends between $0.9 \sigma\left|Y_{i}\right|$ and $1.1 \sigma\left|Y_{i}\right|$ "non-edges" to $Y_{i}$, for any $i \in[r]$,

2. there is at least a $\sigma / 2$-proportion of "non-edges" between any $\tilde{Y}_{i} \subseteq Y_{i}$ and $\tilde{Y}_{j} \subseteq Y_{j}$ with $\left|\tilde{Y}_{i}\right| \geqslant\left|Y_{i}\right| / M$ and $\left|\tilde{Y}_{j}\right| \geqslant\left|Y_{j}\right| / M$, for any distinct $i, j \in[r]$ and any constant $M$. 
Consider an edge-coloring $c$ of $G$ in red and blue and treat the "non-edges" between parts as a third color, "non-edge". We assume that there is no blue induced star on $n$ edges and will prove that there is an induced red copy of $H$.

We apply Szemerédi's Regularity Lemma to $G$ with $\epsilon$ and find a partition of $V(G)$ into an exceptional part $V_{0}$ of size at most $\epsilon|V(G)|$ and parts of equal sizes contained in respective $Y_{i}$ 's, i.e., parts $Y_{i}^{1}, \ldots, Y_{i}^{k_{i}}$, with a total number of parts $k \leqslant M$ such that all but $\epsilon\left(\begin{array}{c}k \\ 2\end{array}\right)$ pairs $Y_{i}^{\ell}, Y_{j}^{m}, i, j \in[r], \ell \in\left[k_{i}\right], m \in\left[k_{j}\right]$, are $\epsilon$-regular in each of the three colors - blue edges of $G$, red edges of $G$, and "non-edges" of $G$. Recall that the proof of the Regularity Lemma allows us to start with an arbitrary partition into a fixed number of parts of equal sizes, and then refine the partition. Thus, we could assume that each part is contained in $Y_{i}$ for some $i$. Note that since the parts are of equal sizes, we have that $k_{1}=\ldots=k_{r-1}=k^{\prime}$ and $k_{r}=\gamma /((r-1)(1+2 \gamma)) k^{\prime}+x k^{\prime}$, with a small term $|x|=O(\epsilon)$.

By the embedding lemma (for example, see Axenovich and Martin [1]), if there are parts $Y_{i}^{f(i)}, i \in[r], f(i) \in\left[k_{i}\right]$, such that all pairs of these parts are $\epsilon$-regular with density at least $\eta$ in both red and in "non-edges", then there is a red induced copy of $H$. Next we shall argue that we can find such a set of $r$ parts. We say it is a good set of $r$ parts.

Consider an auxiliary graph $F$ that is $r$-partite with parts $X_{i}, i \in[r]$ corresponding to $Y_{i}^{\prime}$ 's, such that $\left|X_{i}\right|=k_{i}$ for each $i \in[r]$ and vertices of $X_{i}\left\{x_{i}^{1}, \ldots, x_{i}^{k_{i}}\right\}$ corresponding to parts $Y_{i}^{1}, \ldots, Y_{i}^{k_{i}}$. We say that an edge $e=x_{i}^{\ell} x_{j}^{m}$ is associated with the pair $Y_{i}^{\ell}, Y_{j}^{m}$. Note that $1 / C \leqslant\left|X_{i}\right| /\left|X_{j}\right| \leqslant C$, for $C=(r-1)(1+2 \gamma) / \gamma$. We shall color the edges of $F$ in three colors - yellow, pink, and white as follows. An edge is yellow if the respective pair in $G$ is not $\epsilon$-regular in some of the colors red, blue, or "non-edges". An edge is pink if the respective pair is $\epsilon$-regular, but has density less than $\eta$ in red. All other edges of $F$ are white. Note that a good set of $r$ parts in $G$ correspond to a white $K_{r}$ in $F$. Thus, it is sufficient for us to verify that $F$ satisfies the conditions of Lemma 7 . Let $\epsilon^{\prime}=\frac{(1+3 \gamma)^{2}}{\gamma(1+2 \gamma)} \epsilon 6^{r}$.

Note that since $\sum_{1 \leqslant i \leqslant r}\left|X_{i}\right|=k, \sum_{1 \leqslant i<j \leqslant r}\left|X_{i}\right|\left|X_{j}\right| \geqslant \frac{\gamma(1+2 \gamma)}{(1+3 \gamma)^{2}}\left(\begin{array}{c}k \\ 2\end{array}\right)$. By the Regularity Lemma, the total number of non- $\epsilon$-regular pairs is at most $\epsilon\left(\begin{array}{l}k \\ 2\end{array}\right)$, that is at most $\epsilon \frac{(1+3 \gamma)^{2}}{\gamma(1+2 \gamma)} \sum_{1 \leqslant i<j \leqslant r}\left|X_{i}\right|\left|X_{j}\right|=\epsilon^{\prime} 6^{-r} \sum_{1 \leqslant i<j \leqslant r}\left|X_{i}\right|\left|X_{j}\right|$. Thus, the condition of Lemma 7 on yellow edges is satisfied.

Next we show that the number of pink edges of any vertex of $F$ from part $X_{i}$ to part $X_{j}, j<i$ is at most $\left(\frac{1}{r-1}-\frac{\epsilon^{\prime} C^{3}}{r-1}\right) k_{j}$. If this fails for a vertex $x_{i}^{\ell}$, then we see that in $G$, for any $j, 1 \leqslant j<i$, the number of red edges between $Y_{i}^{\ell}$ and $Y_{j}$ is at most

$$
\left|Y_{i}^{\ell}\right| \cdot\left(\left(\frac{1}{r-1}-\frac{\epsilon^{\prime} C^{3}}{r-1}\right) \eta\left|Y_{j}\right|+\left(1-\frac{1}{r-1}+\frac{\epsilon^{\prime} C^{3}}{r-1}\right)\left|Y_{j}\right|\right) .
$$

Thus there is a vertex in $Y_{i}^{\ell}$ that sends at most $\left(1-(1-\eta)\left(\frac{1}{r-1}-\frac{\epsilon^{\prime} C^{3}}{r-1}\right)\right)\left|Y_{j}\right|$ red edges 
to $Y_{j}$ and thus sends at least $b$ blue edges to $Y_{j}$, where

$$
\begin{aligned}
b & \geqslant\left((1-\eta)\left(\frac{1}{r-1}-\frac{\epsilon^{\prime} C^{3}}{r-1}\right)-1.1 \sigma\right)\left|Y_{j}\right| \\
& \geqslant\left((1-\eta)\left(\frac{1}{r-1}-\frac{\epsilon^{\prime} C^{3}}{r-1}\right)-1.1 \sigma\right)(1+2 \gamma)(r-1) n \\
& \left.=\left[(1-\eta)\left(1-\epsilon^{\prime} C^{3}\right)\right)-1.1(r-1) \sigma\right](1+2 \gamma) n \\
& \geqslant\left[1-\eta-\eta \epsilon^{\prime} C^{3}-\epsilon^{\prime} C^{3}-1.1(r-1) \sigma\right](1+2 \gamma) n \\
& \geqslant[1-\gamma / 2](1+2 \gamma) n \\
& >n .
\end{aligned}
$$

This is a contradiction since we assumed that there is no blue induced star on $n$ edges. The penultimate inequality holds since $1 / 2>\gamma \gg \eta, \gamma \gg(r-1) \sigma$, and $\gamma \gg \epsilon^{\prime}$.

We now see that the conditions of Lemma 7 are satisfied, so there is a white $K_{r}$ in $F$. It corresponds to $r$ parts $Y_{1}^{i_{1}}, \ldots, Y_{r}^{i_{r}}$ in $G$ so that each of the $\left(\begin{array}{l}r \\ 2\end{array}\right)$ pairs formed by these parts is $\epsilon$-regular in all three colors and with red density at least $\eta$. Using property 2 . of $G$, we see that the "non-edges" also have positive density at least $\sigma / 2>\eta$. Thus, by the embedding lemma applied to these $r$ parts, we have an induced red copy of $H$ in $G$.

\subsection{Proof of Theorem 3}

Lemma 8. For any integer $t, t \geqslant 2$, there is an integer $n_{0}$ such that for any $n>n_{0}$, $n+n^{1-\frac{2}{t+1}} \ln ^{-1} n \leqslant \operatorname{IR}\left(K_{t, t}, K_{1, n}\right) \leqslant n+8 n^{1-\frac{1}{t+1}}$.

Proof. For the lower bound we shall take an arbitrary graph $G$ on $n+n^{1-\frac{2}{t+1}}$ vertices and show that there is an edge coloring of $G$ in red and blue with no blue induced star on $n$ edges and no red $K_{t, t}$. Observe first that $G$ contains an independent set of size $n$, call it $A$, otherwise there is no induced $K_{1, n}$ in $G$ and all edges could be colored blue. Let $B=V(G)-A$, let $f(n)=|B|=n^{1-\frac{2}{t+1}} / \ln n$. We shall color all edges in $G[B]$ blue and edges between $A$ and $B$ in red and blue randomly and independently such that each edge is colored red with probability $p$ and blue with probability $(1-p)$. Let $p_{1}$ and $p_{2}$ be the probabilities of bad events - the existence of $K_{t, t}$ with all edges red, and the existence of an induced $K_{1, n}$ with all edges blue, respectively. Then

$$
\begin{aligned}
p_{1} & =\operatorname{Prob}\left(\exists \operatorname{red} K_{t, t}\right) \\
& \leqslant\left(\begin{array}{c}
f(n) \\
t
\end{array}\right)\left(\begin{array}{c}
n \\
t
\end{array}\right) p^{t^{2}} \quad \text { and } \\
p_{2} & =\operatorname{Prob}\left(\exists \text { induced blue } K_{1, n}\right) \\
& \leqslant \operatorname{Prob}\left(\exists K_{1, n-f(n)} \text { with center in } B \text { and leaves in } A\right) \\
& \leqslant f(n)\left(\begin{array}{c}
n \\
n-f(n)
\end{array}\right)(1-p)^{n-f(n)} .
\end{aligned}
$$


Let $p$ be chosen such that $2 f(n) \ln n / n \leqslant p \leqslant \frac{1}{2}(f(n) n)^{-1 / t}$. From the choice of $f(n)$ we see that such a $p$ exists. This gives

$$
\begin{aligned}
p_{1} & \leqslant\left(\frac{e \cdot f(n)}{t}\right)^{t}\left(\frac{e \cdot n}{t}\right)^{t} p^{t^{2}} \\
& \leqslant\left(2(f(n) n)^{t}\left(\frac{1}{2}\right)^{t^{2}}(f(n) n)^{-t}\right. \\
& \leqslant 1 / 2 \quad \text { and } \\
p_{2} & \leqslant f(n)\left(\frac{e n}{f(n)}\right)^{f(n)}(1-p)^{n-f(n)} \\
& \leqslant f(n)\left(\frac{e n}{f(n)}\right)^{f(n)} e^{-p(n-f(n))} \\
& \leqslant f(n)\left(\frac{e n}{f(n)}\right)^{f(n)} e^{-p n / 2)} \\
& <\frac{1}{2} n^{f(n)} e^{-(f(n) \ln n / n) n} \\
& \leqslant \frac{1}{2} n^{f(n)} n^{-f(n)} \\
& =\frac{1}{2}
\end{aligned}
$$

Thus $p_{1}+p_{2}<1$ and there is a coloring that results in no bad events.

For the upper bound, consider $G=K_{f(n), n+f(n)}$, for $f(n)=8 n^{1-\frac{1}{t+1}}=8 n^{\frac{t}{t+1}}$. Consider an arbitrary coloring of $E(G)$ in red and blue. Assume that there is no blue star on $n$ edges and assume that there are no red $K_{t, t}$ 's. Let $A$ be a part of $G$ of size $f(n)$ and $B$ the other part, let $T$ be the number of red stars on $t$ edges with center in $A$. Since there are no blue stars on $n$ edges, each vertex of $A$ is incident to at least $f(n)$ red edges. Thus $T \geqslant|A|\left(\begin{array}{c}f(n) \\ t\end{array}\right)=f(n)\left(\begin{array}{c}f(n) \\ t\end{array}\right)$. On the other hand, for each subset $S$ of $B$ of size $t$, there are at most $t-1$ red stars with leaf set $S$, otherwise there is a red $K_{t, t}$. Thus $T \leqslant\left(\begin{array}{c}|B| \\ t\end{array}\right) t=\left(\begin{array}{c}n+f(n) \\ t\end{array}\right) t$. Putting the inequalities on $T$ together, we see that $f(n)\left(\begin{array}{c}f(n) \\ t\end{array}\right) \leqslant t\left(\begin{array}{c}n+f(n) \\ t\end{array}\right)$. This implies that $f(n)\left(\frac{f(n)}{t}\right)^{t} \leqslant f(n)\left(\begin{array}{c}f(n) \\ t\end{array}\right) \leqslant t\left(\begin{array}{c}n+f(n) \\ t\end{array}\right) \leqslant t\left(\frac{2 n e}{t}\right)^{t}$. Thus $f(n)^{t+1}<t(2 e)^{t} n^{t}$ and $f(n)<8 n^{t /(t+1)}$, a contradiction.

Lemma 9. Let $n$ be sufficiently large. If $H \in\left\{P_{4}, 2 K_{2}\right\}$, then $\operatorname{IR}\left(H, K_{1, n}\right) \leqslant n+$ $2 n \log ^{-1 / 4} n$.

Proof. Let $\epsilon=\log ^{-1 / 4} n$. Let $a=\sqrt{\log n}, b=a^{2}=\log n$, let $X$ be a set of size $b$ and $Y=\left(\begin{array}{c}X \\ b-a\end{array}\right)$, i.e., $Y$ is the set of all subsets of $X$ of size $b-a$. Associate a vertex set $B_{x}$ with each element $x$ of $X$ such that $\left|B_{x}\right|=c, x \in X$, and such that all sets $B_{x}$ are pairwise disjoint and disjoint from $Y$. We choose $c$ such that $c|X|+|Y|=(1+2 \epsilon) n$. 
Let $G$ have vertex set $Y \cup \bigcup_{x \in X} B_{x}$ and edge set $\left\{x^{\prime} y: x^{\prime} \in B_{x}, y \in Y, x \in y\right\}$. I.e., all vertices in $B_{x}$ are adjacent to a set $y$ from $Y$ that contains $x$. One can see that $G$ is obtained by an appropriate blow-up of an incidence graph. We refer to the sets $B_{x}$ as blobs. Note that $|V(G)|=c|X|+|Y|=(1+2 \epsilon) n$. Let $X^{\prime}=\bigcup_{x \in X} B_{x}$. We shall argue that $G \stackrel{\text { ind }}{\longrightarrow}\left(2 K_{2}, K_{1, n}\right)$ and $G \stackrel{\text { ind }}{\longrightarrow}\left(P_{4}, K_{1, n}\right)$.

First we make a few observations about the structure of our graph. We see that

$$
|Y|=\left(\begin{array}{c}
b \\
b-a
\end{array}\right) \leqslant a^{2 a} \leqslant 2^{2 \sqrt{\log n} \log (\sqrt{\log n})} \leqslant 2^{\sqrt{\log n} \log \log n}=o(n \epsilon) .
$$

Thus the total number of vertices in a blown-up part, i.e., in all blobs $B_{x}, x \in X$ is at least $n+2 \epsilon n-o(n \epsilon)$. We see that $c$, the size of each blob is $(n+2 \epsilon n-o(n \epsilon)) / \log n$. Each vertex of $Y$ is not adjacent to $\sqrt{\log n}$ blobs, and adjacent to all vertices of all other blobs. Thus the degree of a vertex from $Y$ in $G$ is at least $(\log n-\sqrt{\log n}) c \geqslant$ $(\log n-\sqrt{\log n})(n+2 \epsilon n-o(n \epsilon)) / \log n \geqslant(1+\epsilon) n$.

Consider a red/blue coloring of the edges of $G$. Assume there is no blue induced star on $n$ edges. Thus each vertex from $Y$ sends at least $\epsilon n$ red edges to $X^{\prime}$. Since each blob has size $c \leqslant n(1+2 \epsilon) /|X|$, we have that each vertex of $Y$ sends a red edge to at least $n_{X}=\frac{\epsilon}{1+2 \epsilon}|X|$ blobs. The total number of red edges is at least $\epsilon n|Y|$, thus there is a vertex, say $x$, in $X^{\prime}$ that is adjacent to a set $Y_{x}$ of at least $\epsilon n|Y| /\left|X^{\prime}\right| \geqslant \frac{\epsilon n|Y|}{(1+2 \epsilon) n}=\frac{\epsilon}{1+2 \epsilon}|Y|$ vertices via red edges. Let $n_{Y}=\frac{\epsilon}{1+2 \epsilon}|Y|$.

Consider $x, Y_{x}$, a set of neighbours of $x$ adjacent to it via red edges, $y_{1} \in Y$, a vertex of $Y$ non-adjacent to $x, y_{2} \in Y_{x}$, and sets $X_{1}$ and $X_{2}, X_{1}, X_{2} \subseteq X$, such that for any $v \in X_{1}$, $y_{1}$ sends a red edge to $B_{v}$ and for any $v \in X_{2}, y_{2}$ sends a red edge to $B_{v}$. If there is a "non-edge" $y^{\prime} u$ between $Y_{x}$ and $X_{1}$ in $I$, we have a red induced $2 K_{2}$ induced by $x, y^{\prime}, y_{1}, x^{\prime}$, where $x^{\prime}$ is a red neighbour of $y_{1}$ in $B_{u}$. If there is a "non-edge" $y^{\prime \prime} u$ between $Y_{x}$ and $X_{2}$ in $I$, we have a red induced $P_{4}$ induced by $y^{\prime \prime}, x, y_{2}, x^{\prime \prime}$, where $x^{\prime \prime}$ is a red neighbour of $y_{2}$ in $B_{u}$.

We have that $\left|X_{i}\right| \geqslant n_{X}=\frac{\epsilon}{1+2 \epsilon}|X|, i=1,2$, and $\left|Y_{x}\right| \geqslant n_{Y}=\frac{\epsilon}{1+2 \epsilon}|Y|$. Thus, it is sufficient for us to check that in $I$ there is a "non-edge" between any subset of $X$ of size $n_{X}$ and any subset of $Y$ of size $n_{Y}$. Assume not, i.e., there is a subset $X^{\prime \prime}$ of $X$ of size $n_{X}$ and $Y^{\prime \prime}$ of $Y$ of size $n_{Y}$ so that $X^{\prime \prime} \cup Y^{\prime \prime}$ induces a complete bipartite graph in $I$.

We shall count the number $t$ of subsets of size $b-a$ containing $X^{\prime \prime}$, recalling that $|X|=b$ and $\left|X^{\prime \prime}\right|=\frac{\epsilon}{1+2 \epsilon} b$ :

$$
t=\left(\begin{array}{c}
b-\left|X^{\prime \prime}\right| \\
b-a-\left|X^{\prime \prime}\right|
\end{array}\right)=\left(\begin{array}{c}
b(1+\epsilon) /(1+2 \epsilon) \\
a
\end{array}\right) .
$$

Since any set in $Y^{\prime \prime}$ is adjacent to all of $X^{\prime \prime}$, we must have that $t \geqslant\left|Y^{\prime \prime}\right|$. We have that

$$
\left|Y^{\prime \prime}\right| \geqslant \frac{\epsilon}{1+2 \epsilon}|Y|=\frac{\epsilon}{1+2 \epsilon}\left(\begin{array}{c}
b \\
b-a
\end{array}\right) .
$$


Thus

$$
\begin{aligned}
\Longrightarrow\left(\begin{array}{c}
b(1+\epsilon) /(1+2 \epsilon) \\
a
\end{array}\right) & \geqslant \frac{\epsilon}{1+2 \epsilon}\left(\begin{array}{c}
b \\
a
\end{array}\right) \\
\Longrightarrow \frac{b(1+\epsilon)}{1+2 \epsilon}\left(\frac{b(1+\epsilon)}{1+2 \epsilon}-1\right) \cdots\left(\frac{b(1+\epsilon)}{1+2 \epsilon}-a+1\right) & \geqslant \frac{\epsilon}{1+2 \epsilon} b \cdots(b-a+1) \\
\Longrightarrow \frac{b(1+\epsilon)}{b} \frac{b(1+\epsilon)-1(1+2 \epsilon)}{b-1} \cdots \frac{b(1+\epsilon)-(a-1)(1+2 \epsilon)}{b-(a-1)} & \geqslant(1+2 \epsilon)^{a-1} \epsilon \\
\Longrightarrow \frac{b}{b} \frac{b-1(1+\epsilon)^{-1}(1+2 \epsilon)}{b-1} \cdots \frac{b-(a-1)(1+\epsilon)^{-1}(1+2 \epsilon)}{b-(a-1)} & \geqslant\left(\frac{1+2 \epsilon}{1+\epsilon}\right)^{a-1} \epsilon .
\end{aligned}
$$

Note that the left hand side of the last inequality is less than 1 . On the other hand, since $\epsilon=1 / \sqrt{a}$, the right hand side is $\left(\frac{1+2 \epsilon}{1+\epsilon}\right)^{a-1} \epsilon \geqslant\left(1+\frac{\epsilon}{1+\epsilon}(a-1)\right) \epsilon \geqslant 1$. This contradiction concludes the proof.

Proof of Theorem 3. The theorem follows from Lemmas 9 and 8.

\section{Conclusions}

In this note, among others, we proved that $\operatorname{IR}\left(H, K_{1, n}\right)$ is asymptotically between $(\chi(H)$ 1) $n$ and $(\chi(H)-1)^{2} n$. For any bipartite graph $H, \chi(H)-1=1$, so both our lower and our upper bound are asymptotically tight. Since the lower bound is the same as for the non-induced Ramsey number $R\left(H, K_{1, n}\right)$, for any $H$, and thus does not explore the induced property, it makes us believe that the lower bound could be improved. Moreover, the upper bound might be close to the true value for all graphs.

\section{Acknowledgements}

The authors thank Casey Tompkins for interesting discussions on $\operatorname{IR}\left(2 K_{2}, K_{1, n}\right)$, Christian Ortlieb for a nice observation on the lower bound in Lemma 4, and Richard Snyder for proofreading parts of the paper. The first author thanks Ryan Martin for conversations about embeddings in multicolor multipartite graphs, Jacob Fox and Stanford University for hospitality. The authors also thank two anonymous referees for several excellent questions and comments as well as constructive suggestions improving the presentation of results.

\section{References}

[1] M. Axenovich and R. Martin, A version of Szemerédi's regularity lemma for multicolored graphs and directed graphs that is suitable for induced graphs, arXiv:1106 . 2871, 2016. 
[2] S. A. Burr, P. Erdős, R. J. Faudree, C. C. Rousseau, R. H. Schelp, Some complete bipartite graph-tree Ramsey numbers, Ann. Discrete Math. 41 (1989), 79-89.

[3] D. Conlon, J. Fox, B. Sudakov, Short proofs of some extremal results II. J. Combin. Theory Ser. B 121 (2016), 173-196.

[4] W. Deuber, A generalization of Ramsey's theorem, Infinite and finite sets (R. Rado A. Hajnal and V. Sós, eds.), vol. 10, North-Holland, 1975, pp. 323-332.

[5] A. Dudek, P. Frankl, V. Rödl, Some recent results on Ramsey-type numbers. Discrete Appl. Math. 161 (2013), no. 9, 1197-1202.

[6] J. Fox, A new proof of the graph removal lemma, Ann. of Math. 174 (2011), 561-579.

[7] J. Fox and B. Sudakov, Induced Ramsey-type theorems, Adv. Math. 219 (2008), 1771-1800.

[8] J. Fox, H. Huang, B. Sudakov, On graphs decomposable into induced matchings of linear sizes. Bull. Lond. Math. Soc. 49 (2017), no. 1, 45-57.

[9] I. Gorgol, A note on lower bounds for induced Ramsey numbers, Discussiones Mathematicae Graph Theory 39 (2019), 647-654.

[10] I. Gorgol, A note on a triangle-free-complete graph induced Ramsey number, Discrete Math. (235) 1-3 (2001), 159-163.

[11] I. Gorgol, Planar and Induced Ramsey Numbers (in Polish), PhD thesis, Adam Mickiewicz University, Poznan, 2000.

[12] F. Harary, J. Nešetřil, V. Rödl, Generalized Ramsey theory for graphs. XIV. Induced Ramsey numbers, Graphs and other combinatorial topics (Prague, 1982) 59 (1983), 90-100.

[13] Y. Kohayakawa, H. J. Prömel, V. Rödl, Induced Ramsey numbers, Combinatorica 18 (1998), 373-404.

[14] A. Kostochka and N. Sheikh, On the induced Ramsey number $\operatorname{IR}\left(P_{3} ; H\right)$, Topics in discrete mathematics 26 (2006), 155-167.

[15] T. Kövári, V.T. Sós, P. Turán, On a problem of K. Zarankiewicz, Colloq. Math. 3 (1954), 50-57.

[16] T. D. Parsons, Ramsey graphs and block designs, Trans. Am. Math. Soc. 209 (1975), $33-44$.

[17] F. Ramsey, On a problem in formal logic, Proceedings of London Mathematical Society, (1927), 30: 264-286.

[18] M. Schaefer and P. Shah, Induced graph Ramsey theory, Ars Combin. 66 (2003), $3-21$.

[19] D. West, Introduction to graph theory, Prentice Hall, Inc., Upper Saddle River, NJ, 1996. xvi+512 pp. 